Ambiente \& Água - An Interdisciplinary Journal of Applied Science
ISSN 1980-993X - doi:10.4136/1980-993X
www.ambi-agua.net
E-mail: ambi.agua@gmail.com

\title{
Análise de índices biofísicos a partir de imagens TM Landsat 5 em paisagem heterogênea no Sudoeste da Amazônia
}

\author{
doi:10.4136/ambi-agua.1663
}

Received: 14 May 2015; Accepted: 25 Aug. 2015

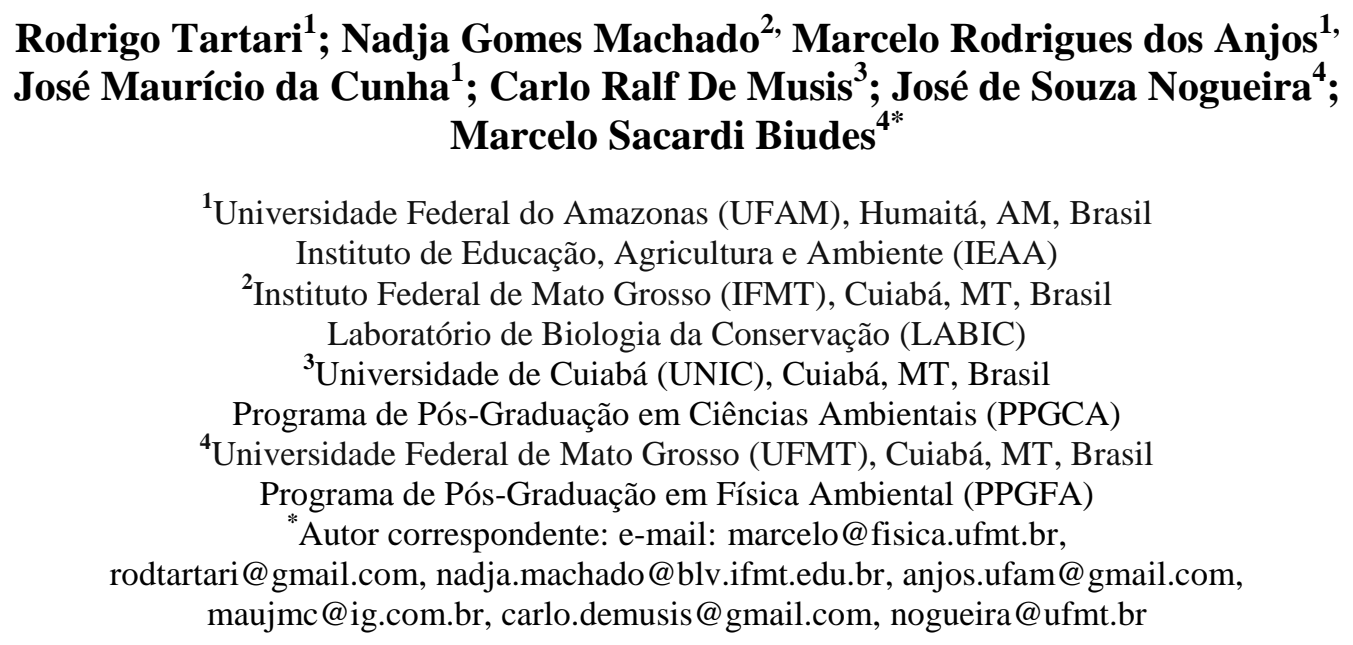

\section{RESUMO}

O sudoeste da Amazônia Brasileira é composto por um mosaico de paisagem com transição de campos naturais e floresta ombrófila densa. A bacia do rio Puruzinho, localizada no "Arco do Desmatamento" no interflúvio Purus-Madeira, tem passado por mudança no uso e cobertura da terra. Assim, o objetivo deste estudo foi avaliar a variação espacial de índices biofísicos de uma paisagem heterogênea na bacia hidrográfica do rio Puruzinho. $O$ índice de vegetação da diferença normalizada (NDVI), índice de área foliar (IAF), temperatura da superfície, albedo e saldo de radiação da superfície (Rn) foram estimados pelo SEBAL (Surface Energy Balance Algorithm for Land) a partir de imagens TM Landsat 5, e analisados sobre floresta densa, campos naturais, área desmatada e área urbana de Humaitá-AM. Todos os índices biofísicos foram significativamente diferentes, considerando o uso e cobertura da terra. Os índices biofísicos apresentaram padrão inverso entre a área urbana e a floresta densa, com menor NDVI, IAF e saldo de radiação e maior temperatura e albedo da superfície na área urbana. Portanto, as variáveis estimadas pelo SEBAL foram adequadas para avaliar o efeito das mudanças no uso do solo sobre as variáveis estimadas e distinguir áreas antropizadas de áreas naturais.

Palavras-chave: análise espacial, bacia amazônica, balanço de radiação, desmatamento.

\section{Evaluation of biophysical indices from TM Landsat 5 images in heterogeneous landscape in Southwestern Amazon}

\section{ABSTRACT}

The southwest area of the Brazilian Amazon is composed of a landscape mosaic with transition grasslands and tropical rain forest. The basin of the Puruzinho River, located in the 
"Arc of Deforestation" in the Purus-Madeira interfluve, has undergone changes in land use and land cover. The objective of this study was to evaluate the spatial variation of biophysical indices of a heterogeneous landscape in the Basin of Puruzinho River. The normalized difference vegetation index (NDVI), leaf area index (LAI), surface temperature, surface albedo and surface net radiation (Rn) were estimated by SEBAL (Surface Energy Balance Algorithm for Land) from TM Landsat 5 images, and analyzed on areas of dense forest, grasslands, deforested area and urban area of Humaitá-AM. All biophysical indices were significantly different considering the land use and land cover. The biophysical indices showed an opposite pattern between the urban area and the dense forest, with lower NDVI, IAF and net radiation and higher surface temperature and surface albedo in the urban area. Therefore, the variables estimated by SEBAL were adequate to assess the effect of land use changes on the estimated variables and to distinguish disturbed areas from natural areas.

Keywords: Amazonia, deforestation, radiation balance, spatial analysis.

\section{INTRODUÇÃO}

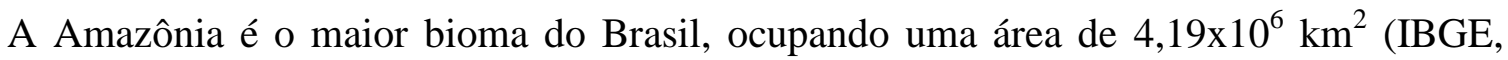
2004). Esse bioma possui grande biodiversidade e particularidades físicas naturais de relevo e solo, apresentando um mosaico de 23 ecorregiões naturais (Brasil, 1978). Dentre elas, a ecorregião do interflúvio Purus-Madeira apresenta-se com expressivos contrastes de vegetação, representadas por manchas de savanas, as quais ocupam dimensões significativas em ecótonos com áreas de floresta densa, no sudoeste da Amazônia Brasileira (Soares-Filho et al., 2005).

A ecorregião do interflúvio Purus-Madeira se encontra na faixa do "Arco do Desmatamento", em cenário de desenvolvimento e mudanças no uso e cobertura da terra devido à instalação das UHE de Santo Antônio e Jirau, reconstrução de rodovias, atividades de exploração de recursos minerais e produtos florestais, agropecuária e urbanização (Fearnside, 2015). Essas atividades representam os principais aspectos indicadores de desenvolvimento e riscos de alteração da paisagem. A substituição de áreas nativas por pastagem e área urbana alteram significativamente a temperatura e umidade relativa do ar e os balanços de radiação e energia da superfície em escala local e regional (Biudes et al., 2015).

O monitoramento ambiental em escala regional pode ser realizado a partir de técnicas de sensoriamento remoto, as quais permitem analisar a relação entre padrões espaciais da vegetação e as mudanças no balanço de radiação e dos fluxos de energia da superfície (Fausto et al., 2014). O algoritmo SEBAL (Surface Energy Balance Algorithm for Land) possibilita estimar o balanço de energia da superfície (Bastiaanssen, 1998), a partir de índices biofísicos da superfície, como o Índice de Vegetação da Diferença Normalizada (NDVI), albedo, temperatura e saldo de radiação da superfície. Dessa forma, o SEBAL pode ser aplicável ao monitoramento e detecção de mudanças no uso e cobertura da terra, assim como possíveis impactos sobre as variáveis ambientais em escala regional. Alteração do uso e cobertura da terra, seja para estabelecimento de área agrícolas ou urbana, aumentam o albedo e a temperatura da superfície, bem como a redução de índices de vegetação e o saldo de radiação da superfície (Oliveira et al., 2012; Nascimento et al., 2014). Este algoritmo também permite análise espacial, e pode ser indicada para estudos de zoneamentos socioambientais e projetos urbanísticos ligados ao conforto térmico urbano (Gartland, 2010).

Diante das mudanças de uso e cobertura do solo ocorridas na região Amazônica e a possibilidade de seu diagnóstico com o uso do sensoriamento, o objetivo deste estudo foi avaliar a variação espacial de índices biofísicos como os índices de vegetação, albedo, temperatura e saldo de radiação da superfície de uma paisagem heterogênea na bacia hidrográfica do rio Puruzinho. 


\section{MATERIAL E MÉTODOS}

\section{1. Área de Estudo}

A área de estudo foi a bacia do rio Puruzinho que está localizada no interflúvio PurusMadeira, Sudoeste da Amazônia Brasileira (Figura 1). Essa bacia pertence às formações dos Campos Puciari de Humaitá-AM que abrangem parte do Sul do Amazonas e Norte de Rondônia (Vidotto et al., 2007) e se caracteriza por diferentes usos do solo, com manutenção de sua vegetação nativa, o que não tem sido comum em outras regiões do Brasil. A bacia do rio Puruzinho, inserida em zona de transição entre campos e florestas, possui uma área de $1146 \mathrm{~km}^{2}$ composta por $63,4 \%$ de floresta densa, $23,2 \%$ de campos naturais, e $0,5 \%$ de espelho d'água que se refere principalmente ao lago do Puruzinho. Além disso, apresenta áreas antropizadas por agropecuária, extrativismo, rodovias (BRs 230 e 319), ocupação urbana e área desmatada, num total de 12,9\% da área da bacia (Carvalho et al., 2014). Sendo assim, foram utilizadas cinco classes: floresta densa (FD), campos naturais (CN), área desmatada (DE) e área urbana (AU) do município de Humiatá-AM e espelho d’água (EA).

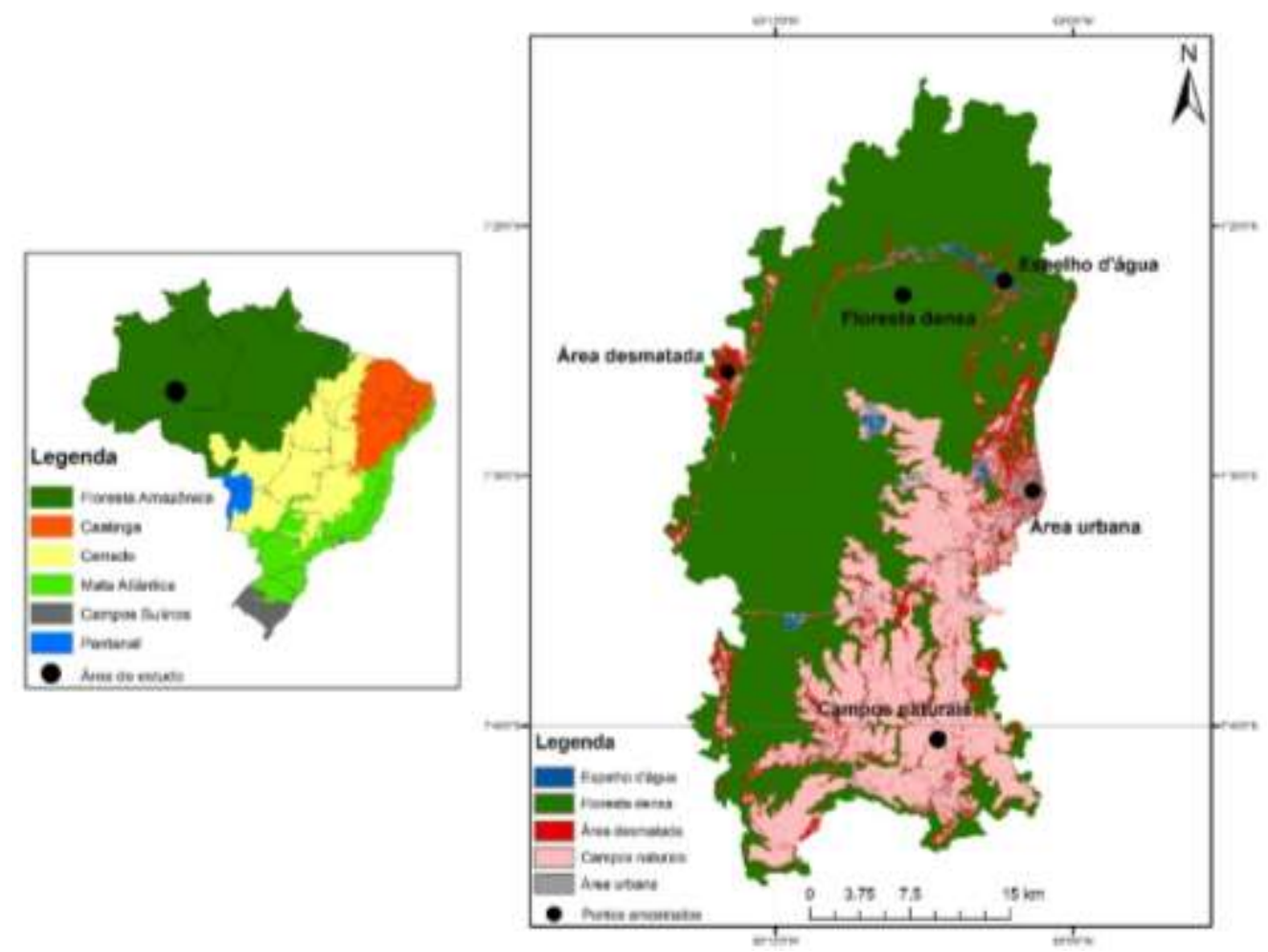

Figura 1. Localização da bacia do Rio Puruzinho no Sudoeste da Amazônia Brasileira, classes de uso e cobertura da terra e pontos de extração dos índices biofísicos.

\subsection{Estimativas de índices biofísicos pelo SEBAL}

A área da bacia foi delimitada com base em imagem do SRTM (Radar Shuttle Radar Topography Mission), com resolução espacial de 90 metros (Farr et al., 2007), adquirida em http://www.dsr.inpe.br/topodata/index.php. Os índices biofísicos foram estimados pelo algoritmo SEBAL (Surface Energy Balance Algorithm for Land) (Bastiaanssen, 1998), a partir de uma imagem corrigidas para efeitos da atmosfera geradas pelo sensor Thematic Mapper (TM), acoplado ao satélite Landsat 5, adquiridas em http://earthexplorer.usgs.gov/.

A imagem analisada nesse trabalho corresponde à passagem do Landsat 5 sobre a área de estudo em dia de céu claro às 10h09 (hora local) no dia 7/05/2011 (Dia Juliano = 127), na órbita 232, ponto 65 . A radiação solar diária foi calculada por meio da insolação e a 
transmissividade atmosférica foi calculada com base na temperatura e umidade relativa do ar, medidos na estação meteorológica (código OMM 81890) de Humaitá ( $7^{\circ} 55^{\prime \prime}$ S e $63^{\circ} 07^{\prime \prime} \mathrm{O}$ ) operada pelo INMET.

Os índices biofísicos foram calculados segundo o detalhamento descrito em Fausto et al. (2014). Para tanto, foram seguidas as seguintes etapas: (i) recorte das imagens obtidas do TM Landsat 5 da área estudo; (ii) empilhamento das 7 bandas do TM Landsat 5 em um único arquivo de imagem; (iii) classificação supervisionada do uso e cobertura da terra; e (iv) cálculo do índice de vegetação da diferença normalizada (NDVI - Normalized Difference Vegetation Index), Índice de Área Foliar (IAF), albedo da superfície ( $\alpha$ ), temperatura radiométrica da superfície (Ts) e saldo de radiação da superfície (Rn).

\subsection{Análises estatísticas}

Foram extraídos os valores de 9 pixel em torno de 3 pontos por tipo de classe, totalizando 2.43 ha. As médias dos índices biofísicos por classe e o intervalo de confiança de $\pm 95 \%$ das estimativas foram calculadas por bootstrapping de 1000 iterações das reamostragens aleatórias com substituição (Efron e Tibshirani, 1993). A correlação de Spearman foi utilizada para avaliar a força e direção da correlação entre as variáveis estudadas.

\section{RESULTADOS E DISCUSSÃO}

Não houve diferença significativa nos valores de NDVI e o IAF entre área desmatada e campos naturais, mas houve diferença significativa do NDVI entre as outras classificações (Figura 2C e 2D). O NDVI da floresta densa $(0,72)$ foi $85,3 \%$ maior do que o da área urbana $(0,39)$. As áreas de corpos d'água apresentaram valores de NDVI próximos de zero (Figura 2A). O IAF da floresta densa $\left(1,1 \mathrm{~m}^{2} \mathrm{~m}^{-2}\right)$ foi $117 \%$ maior do que a área urbana e os campos naturais $\left(0,1 \mathrm{~m}^{2} \mathrm{~m}^{-2}\right)$. As áreas escuras formadas por corpos d'água apresentaram valores de NDVI menores que zero (Figura 2A) e IAF próximos de zero (Figura 2B).

As estimativas de NDVI da área de estudo estão de acordo com outros estudos desenvolvidos em regiões tropicais. Nascimento et al. (2014) encontraram valores de NDVI acima de 0,40 em áreas verdes, entre 0,01 e 0,30 em aglomerados urbanos, e valores próximos de zero em corpos hídricos em Mossoró-RN. Outros estudos corroboram as estimativas de IAF da área de estudo ao apontar que o IAF em regiões tropicais pode variar de 0,5 a $3 \mathrm{em}$ pastagem na bacia Amazônica em Rondônia (Zanchi et al., 2009), de 6 a 8 em floresta de transição Amazônia-Cerrado, e de 2,5 a 4,5 em floresta inundável no Pantanal em Mato Grosso (Biudes et al. 2014), e de 2 a 4 em área urbana em Humaitá, AM (Lopes, 2013).

Destaca-se que, os maiores valores de NDVI e IAF indicam maior biomassa (Fausto et al., 2014). A variação espacial do NDVI com menores valores em áreas antropizadas e maiores valores em área com vegetação foi semelhante à encontrada por Gomes et al. (2013), Andrade e Corrêa (2014), Fausto et al. (2014), e Nascimento et al. (2014). A variação espacial do IAF também com menores valores em áreas antropizadas e maiores valores em área com vegetação de floresta foi semelhante aos valores encontrados por Fausto et al. (2014).

As áreas mais claras apresentaram maiores valores de albedo que correspondem às áreas com impacto antrópico como área urbana, área desmatada e rodovias (Figura 3A). O albedo da superfície foi significativamente diferente entre as classes analisadas, com maiores valores na área urbana (21\%), e menores valores no espelho d'água (4\%) (Figura 3C).

Maiores valores de albedo da superfície em áreas antropizadas e menores em área com vegetação densa também foram encontrados por outros autores. Liberato (2011) obteve albedo de $11 \%$ em floresta Amazônica e 13\% em áreas de pastagem próximo de Ji-ParanáRO; Von Randow et al. (2004) obtiveram albedo de 13\% em floresta Amazônica e 22\% em pastagem na REBIO Jaú-RO; e Querino et al. (2006) obtiveram albedo entre 13 e $15 \%$ em 
floresta Amazônica e entre 17 e $22 \%$ em pastagem. O albedo do espelho d'água foi próximo ao encontrados por Liberato (2011), mas diferente do valor próximo de zero encontrado por Fausto et al. (2014) no rio Cuiabá, MT. Isto pode indicar alta carga de sedimentos na água da área de estudo, decorrente de processos erosivos da bacia e fluxos de sedimentos oriundos do rio Madeira (Fearnside, 2015).
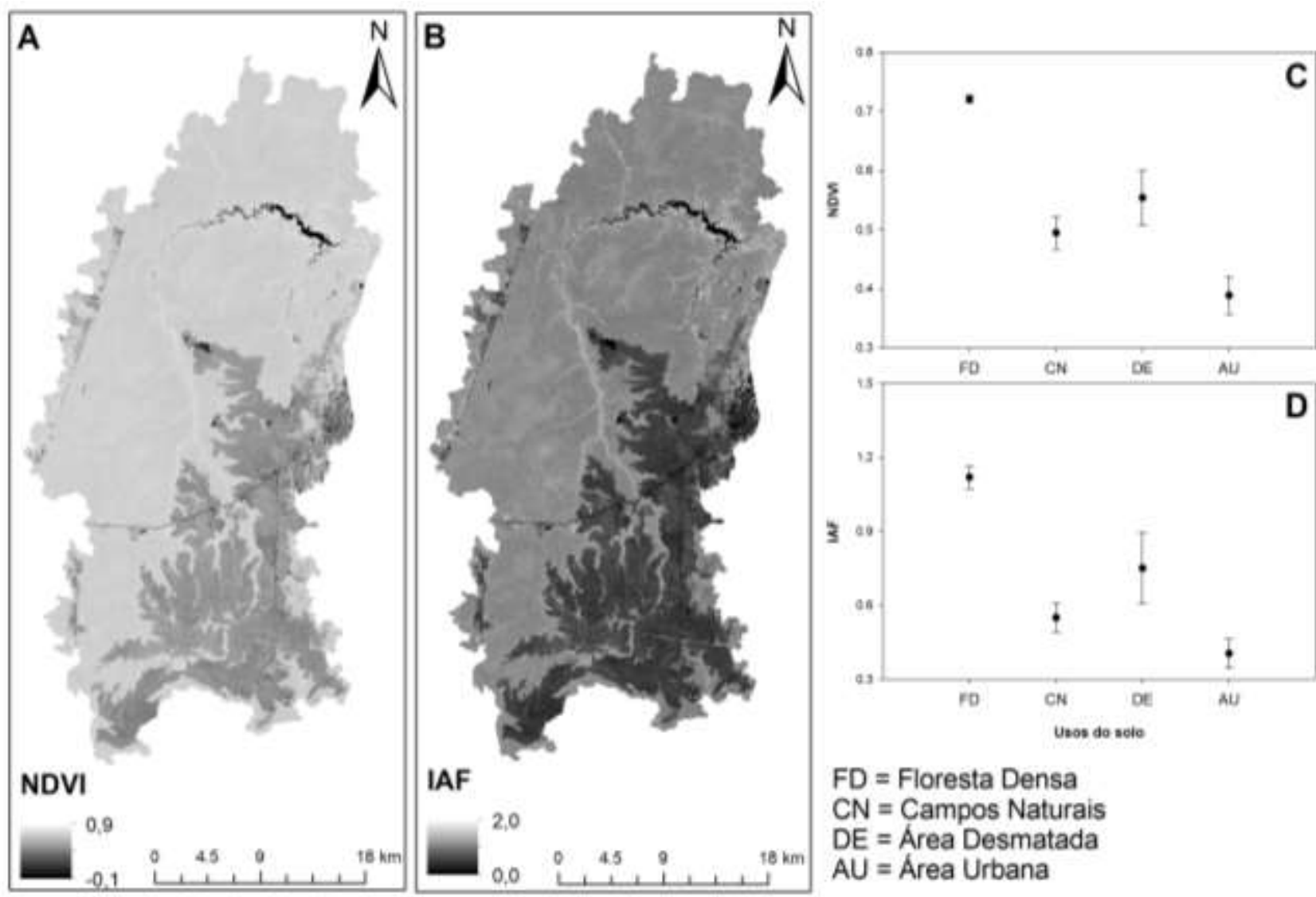

Figura 2. Mapa do índice de vegetação da diferença normalizada (NDVI) (A) e do índice de área foliar (IAF) (B) e valores do NDVI (C) e IAF (D) nos diferentes usos do solo na bacia do Rio Puruzinho, Amazonas, Brasil. As barras representam $\pm 95 \%$ de intervalo de confiança.

Não houve diferença significativa nos valores de temperatura da superfície durante a passagem do satélite entre área desmatada $\left(23,7^{\circ} \mathrm{C}\right)$ e campos naturais $\left(25,1^{\circ} \mathrm{C}\right)$. No entanto, houve diferença significativa entre as outras classificações, com temperatura da superfície na área urbana $28,7 \%$ maior que na floresta densa $\left(20,8^{\circ} \mathrm{C}\right)$ (Figura 3D). As maiores temperaturas ocorreram nas áreas mais claras que correspondem às áreas antropizadas por área urbana, área desmatada e rodovias (Figura 3B). Algumas manchas mais "claras" estão localizadas onde existem corpos hídricos, indicando o efeito da antropização às suas margens. Ressalta-se que a banda termal do TM Landsat 5 tem resolução espacial de $120 \mathrm{~m}$, enquanto que as demais bandas têm resolução espacial de $30 \mathrm{~m}$. Dessa forma, a antropização das margens dos rios tem maior efeito na estimativa da temperatura da superfície que os corpos d’água.

O saldo de radiação da superfície foi significativamente diferente entre as classes analisadas, com maiores valores no espelho d'água $\left(595,5 \mathrm{~W} \mathrm{~m}^{-2}\right)$ e menores valores em área urbana $\left(455,7 \mathrm{~W} \mathrm{~m}^{-2}\right.$ ) (Figura $\left.4 \mathrm{~A}\right)$. As áreas mais escuras correspondem às áreas antropizadas e apresentaram os menores valores de saldo de radiação da superfície (Figura 4B). 

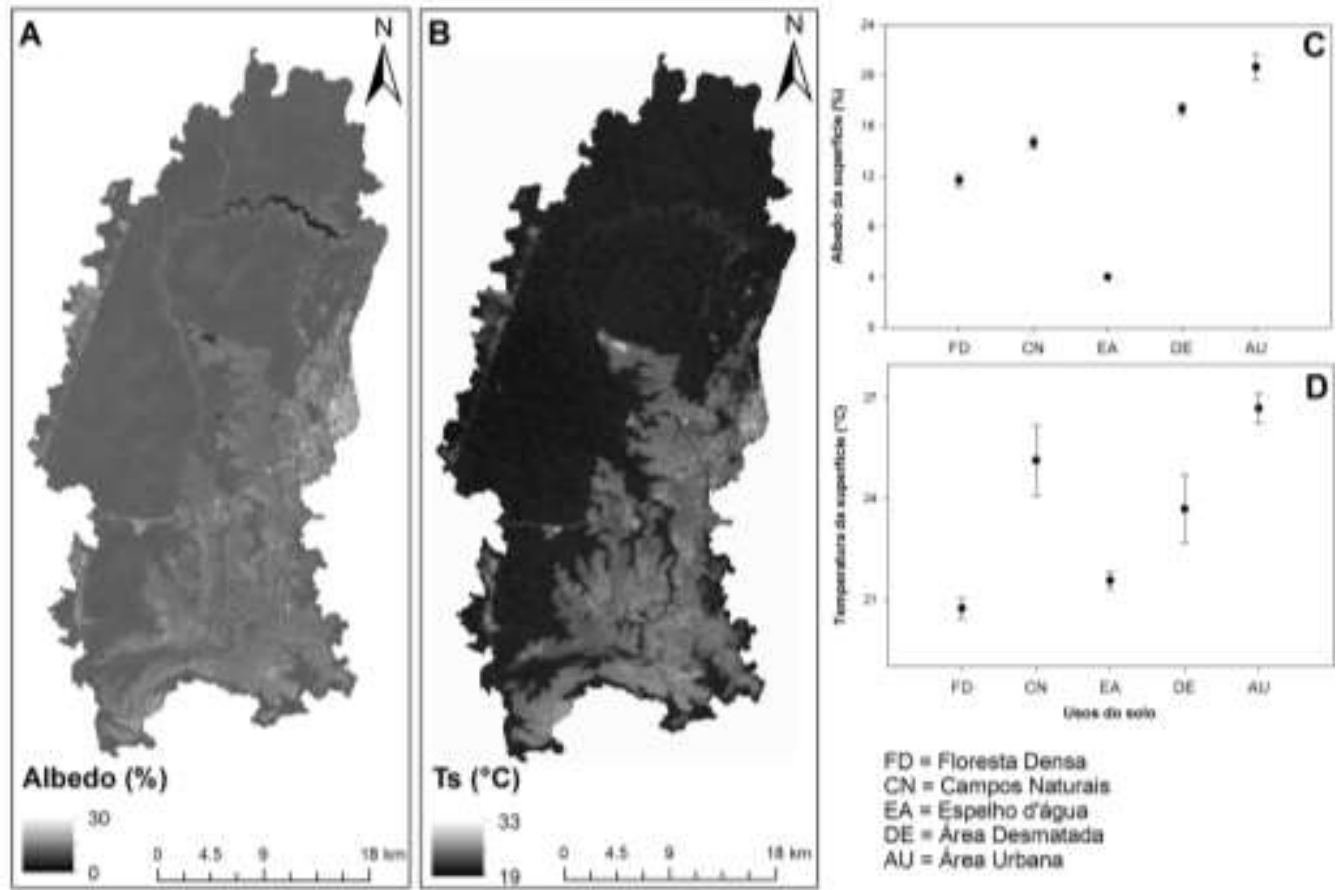

Figura 3. Mapa do albedo (A) e da temperatura da superfície (B), e valores do albedo (C) e valores da temperatura da superfície (D) em diferentes usos do solo na bacia do Rio Puruzinho, Amazonas, Brasil. As barras representam $\pm 95 \%$ de intervalo de confiança.
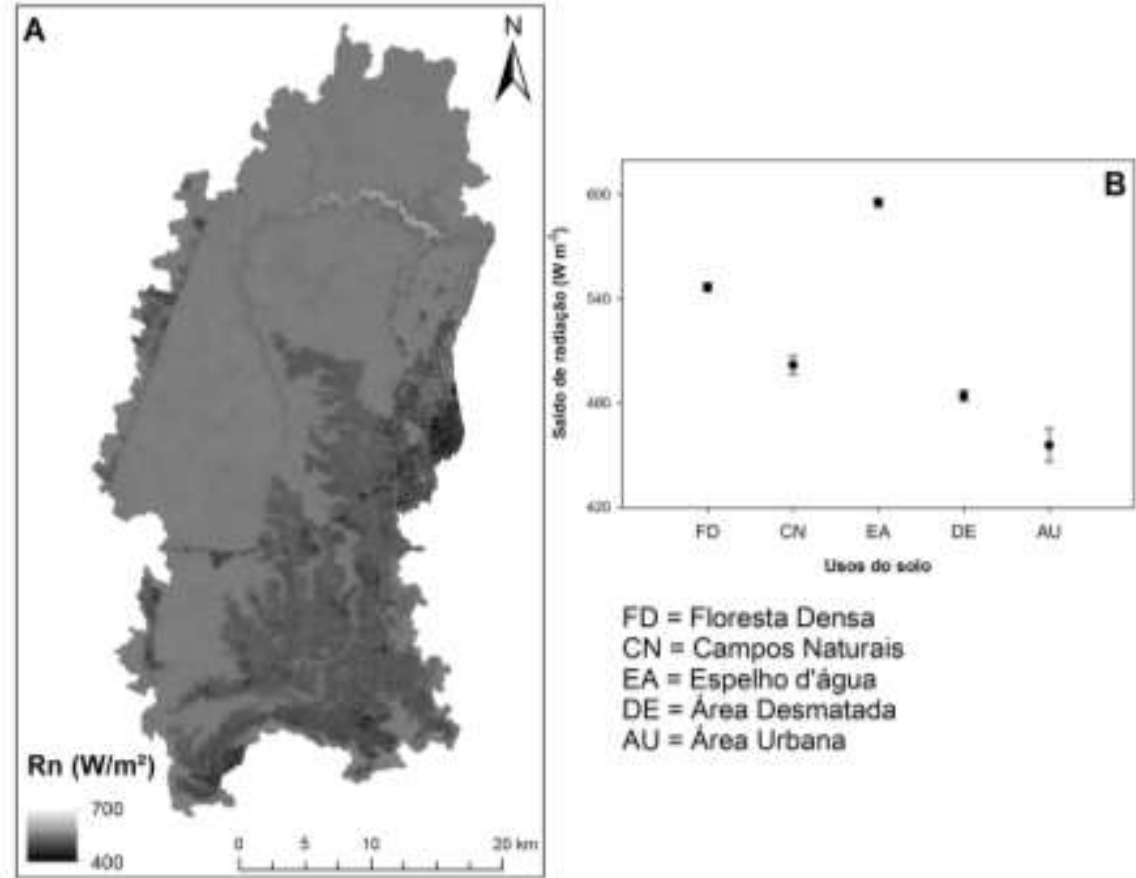

FD $=$ Floresta Densa

$\mathrm{CN}=$ Campos Naturais

$E A=$ Espelho d'água

$\mathrm{DE}=$ Área Desmatada

$\mathrm{AU}=$ Area Urbana

Figura 4. Mapa (A) e valores (B) do saldo de radiação da superfície $(\mathrm{Rn})$ em diferentes usos do solo na bacia do Rio Puruzinho, Amazonas, Brasil. As barras representam $\pm 95 \%$ de intervalo de confiança.

A substituição de floresta nativa densa por áreas antropizadas provocou aumento na temperatura da superfície (Figura 5; Tabela 1). A estimativa dos índices biofísicos na área urbana apresentou padrão inverso ao da floresta densa, uma vez que apresentou maior 
temperatura e albedo da superfície, e menor saldo de radiação, NDVI e IAF (Figura 5; Tabela 1). O comportamento dessas variáveis na área urbana pode ser explicado pelos balanços de radiação e energia que são modificados com a substituição de vegetação por áreas construídas.

Tabela 1. Matriz de correlação de Spearman do saldo de radiação da superfície (Rn), a temperatura da superfície (Ts), Índice de Vegetação da Diferença Normalizada (NDVI), Índice de Área Foliar (IAF) e albedo da superfície na Bacia do rio Puruzinho, Amazonas, Brasil.

\begin{tabular}{lcccc}
\hline Variável & $\mathrm{Rn}\left(\mathrm{W} \mathrm{m}^{-2}\right)$ & $\mathrm{Ts}\left({ }^{\circ} \mathrm{C}\right)$ & $\mathrm{NDVI}$ & $\mathrm{IAF}\left(\mathrm{m}^{2} / \mathrm{m}^{2}\right)$ \\
\hline Ts $\left({ }^{\circ} \mathrm{C}\right)$ & $-0.58^{* *}$ & & & \\
NDVI & $0.38^{* * *}$ & $-0.61^{* *}$ & & \\
IAF & $0.30^{* *}$ & $-0.63^{* *}$ & $0.90^{* *}$ & \\
Albedo & $-0.82^{* *}$ & $0.39^{* *}$ & $-0.33^{* *}$ & $-0.23^{* *}$ \\
\hline
\end{tabular}

** p-valor $>0,01$.

Em geral, a diferença no saldo de radiação da superfície entre as diferentes classes analisadas nesse estudo está ligada à estrutura e/ou falta da vegetação, à disponibilidade hídrica da superfície e às propriedades térmicas da superfície (Oke et al., 1999; Christen e Vogt, 2004). As relações entre os índices biofísicos analisados nesse estudo são bem documentadas em estudos envolvendo efeitos da conversão entre floresta e pastagem (Liberato, 2011; Gomes et al., 2013; Andrade e Corrêa, 2014; Fausto et al., 2014; Nascimento et al., 2014). No entanto, poucos estudos se referem a efeitos da conversão de área nativa em urbana.

A diferença observada no albedo da superfície na floresta e nos campos foi devido aos processos de absorção, reflexão e transmissão que ocorre entre a radiação incidente e a superfície estudada (Pereira et al., 2007). A radiação incidente sobre campo e/ou área urbana é pouco utilizada pela vegetação para os processos de evapotranspiração e fotossíntese em decorrência de seu baixo índice de área foliar e/ou à grande extensão de áreas de concreto, o que aumenta o seu espalhamento devido às múltiplas reflexões. Todavia, a maior parte da radiação incidente é absorvida pela floresta, pois o dossel arbóreo apresenta refletância relativamente baixa devido ao uso dessa energia por pigmentos fotossintetizantes presentes nas folhas e às múltiplas reflexões no interior do dossel (Ponzoni, 2012).

Mesmo apresentando menor albedo, e consequentemente maior absorção da radiação incidente, a floresta apresenta menor temperatura radiométrica em função da maior disponibilidade hídrica e à maior distribuição dessa energia radiativa para os processos de evapotranspiração e fotossíntese. Já as áreas de campo apresentam distribuem, prioritariamente, a energia disponível para os processos de aquecimento do ar e do solo, devido à baixa densidade de vegetação que tem baixa capacidade de armazenamento de água no interior do dossel (Biudes et al., 2009; 2015).

As áreas urbanas têm complexas trocas de energia, massa e momentum, devido aos diversos elementos que compõem sua superfície, pois o tecido urbano consiste de uma estrutura complexa de blocos de construção, que produzem um mosaico de superfícies iluminadas e sombreadas e, consequentemente afetam o albedo (Frey et al., 2007). A radiação de onda curta incidente não é igualmente distribuída dentro das camadas do dossel urbano devido ao sombreamento e exposição dos edifícios que provocam divergência vertical da densidade de fluxo de radiação (Christen e Vogt, 2004). O albedo elevado na área urbana está associado à superfícies de cores lisas, secas, e claras, enquanto que um albedo baixo está associado à superfícies ásperas, úmidas, e escuras (Silva et al., 2011). 

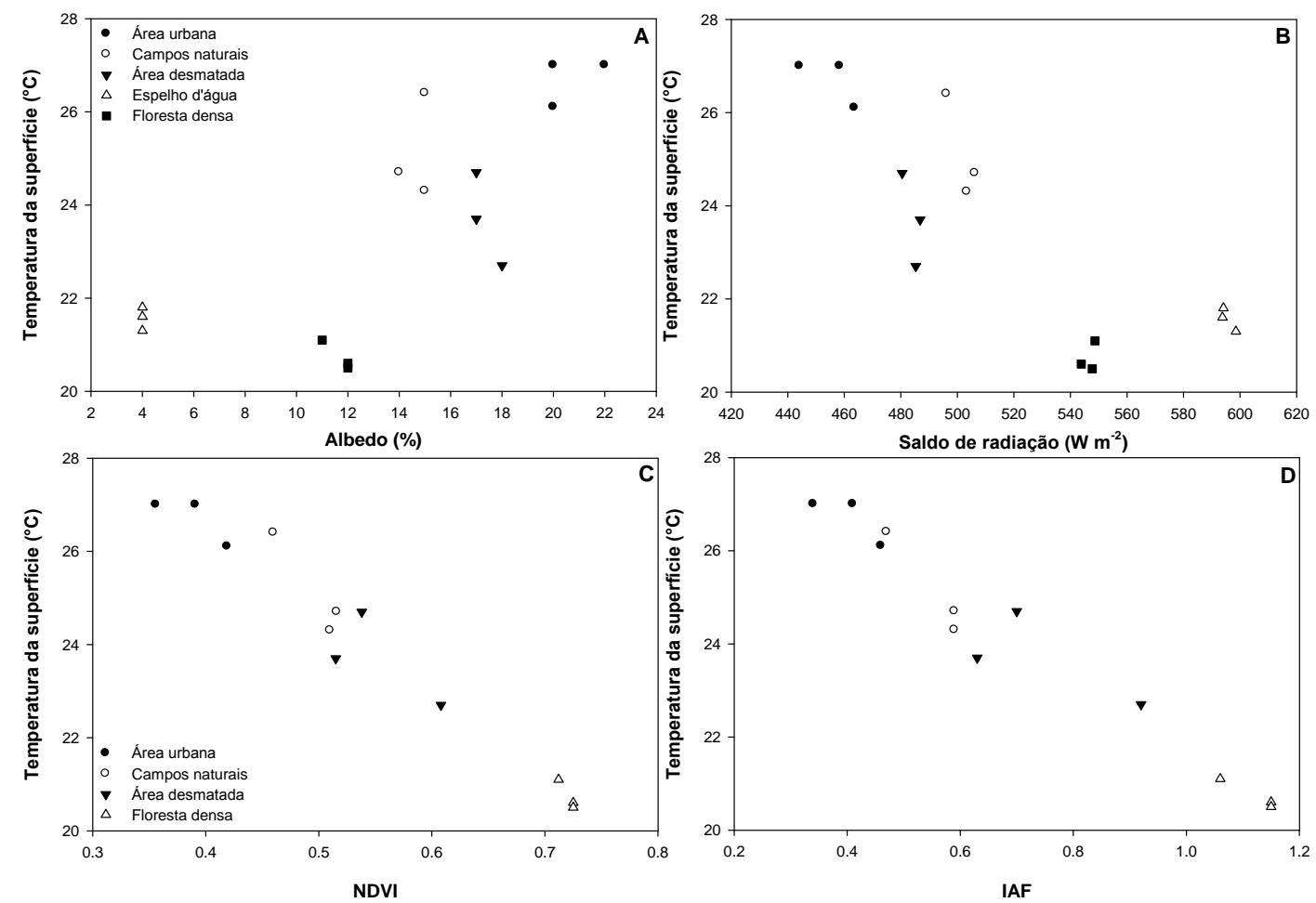

Figura 5. Relação entre temperatura da superfície e albedo (A), saldo de radiação (B), índice NDVI (C), e índice de área foliar (IAF) (D) nos diferentes usos do solo na bacia do Rio Puruzinho, Amazonas, Brasil.

A radiação de onda longa incidente aumenta em áreas urbanas, quer devido ao aumento do calor da atmosfera urbana e/ou da emissividade atmosférica reforçada provocada pela presença de partículas e gases poluentes (Estournel et al., 1983). As partículas em uma atmosfera poluída que absorvem e dispersam a radiação de ondas curtas, também absorvem a radiação de onda longa, criando uma perda de calor na superfície e aquecimento da camada poluída, mas também emitem radiação de onda longa, que arrefece a camada poluída (Christen e Vogt, 2004). Por outro lado, a radiação de onda longa reemitida pela superfície é controlada pela temperatura da própria superfície (Frey et al., 2007). Áreas urbanas apresentam maior magnitude de radiação de onda longa reemitida pela superfície que áreas rurais, devido à maior temperatura da superfície e/ou emissividade diferente (Christen e Vogt, 2004). Destaca-se ainda, que as áreas urbanas possuem maior capacidade de armazenar calor e menor quantidade de energia destinada à evapotranspiração (Grimmond e Oke, 1999). Assim, a maior parte da energia disponível é empregada ao aquecimento do ar e do solo em áreas urbanas. Como o ambiente urbano é dominado por calor sensível, grandes quantidades de calor são armazenadas no tecido urbano durante o dia e são liberadas durante a noite (Oke et al., 1999).

\section{CONCLUSÃO}

Os índices biofísicos apresentaram padrão inverso entre a área urbana e a floresta densa, uma vez que a área urbana apresentou maior albedo e temperatura da superfície, e menores valores de NDVI, IAF e saldo de radiação. Portanto, as estimativas de NDVI, IAF, albedo e temperatura da superfície e saldo de radiação pelo SEBAL foram adequados para distinguir áreas antropizadas de áreas naturais e avaliar o efeito das alterações de uso e ocupação do solo sobre as variáveis estudadas. 


\section{AGRADECIMENTOS}

A pesquisa foi apoiada pela Universidade Federal de Mato Grosso (UFMT), Programa de Pós Graduação em Física Ambiental (PPGFA/IF/UFMT), e Coordenação de Aperfeiçoamento de Pessoal do Ensino Superior (CAPES - Bolsas, Processo n ${ }^{\circ}$ 9750/13-4 e no 9768/13-0), e financiada pela Fundação de Amparo à Pesquisa do Estado de Mato Grosso (FAPEMAT PRONEM 2014, processo no 561397/2014).

\section{REFERENCIAS}

ANDRADE, S. C. P.; CORRÊA, J. A. J. Estimativa do saldo de radiação instantâneo à superfície para a cidade de Santarém-PA, através de imagens do Landsat 5-TM. Revista Brasileira de Geografia Física, v. 7, n. 4, p. 653-661, 2014.

BASTIAANSSEN, W. G. M.; MENENTI, M.; FEDDES, R. A.; HOLSLAG, A. A. M. A Remote Sensing Surface Energy Balance Algorithm for Land (SEBAL) - Formulation. Journal of Hydrology, v. 212-213, p. 198-212, 1998. http://dx.doi.org/10.1016/S00221694(98)00253-4

BIUDES, M. S.; CAMPELO JUNIOR, J. H.; NOGUEIRA, J. S.; SANCHES, L. Estimativa do balanço de energia em cambarazal e pastagem no norte do Pantanal pelo método de razão de Bowen. Revista Brasileira de Meteorologia, v. 24, n. 2, p. 135-143, 2009. http://dx.doi.org/10.1590/S0102-77862009000200003

BIUDES, M. S.; MACHADO, N. G.; DANELICHEN, V. H. M.; SOUZA, M. C.; VOURLITIS, G. L.; NOGUEIRA, J. S. Ground and remote sensing-based measurements of leaf area index in a transitional forest and seasonal flooded forest in Brazil. International Journal of Biometeorology, v. 58, p. 1181-1193, 2014. http://dx.doi.org/10.1007/s00484-013-0713-4

BIUDES, M. S.; VOULITIS, G. L.; MACHADO, N. G.; DE ARRUDA, P. H. Z.; NEVES, G. A. R.; LOBO, F. A. et al.Patterns of energy exchange for tropical ecosystems across a climate gradiente in Mato Grosso, Brazil. Agricultural and Forest Meteorology, v. 202, p. 112-124, 2015. http://dx.doi.org/10.1016/j.agrformet.2014.12.008

BRASIL. Departamento Nacional da Produção Mineral. Projeto Radambrasil. Folha SB 20 Purus, 1978. $566 \mathrm{p}$.

CARVALHO, R. R.; TARTARI, R.; RADMANN, V.; PAGANI, C. H. P. Monitoramento da vazão em rios da região sul do Amazonas. Revista EDUCAmazônia, v. 12, n. 1, p. 8$27,2014$.

CHRISTEN, A.; VOGT, R. Energy and radiation balance of a central European city. International Journal of Climatology, v. 24, p. 1395-1421, 2004. http://dx.doi.org/10.1002/joc.1074

EFRON, B.; TIBSHIRANI, R. J. An introduction to the bootstrap. New York: Chapman \& Hall, 1993. 443 p.

ESTOURNEL, C.; VEHIL, R.; GUEDALIA, D.; FONTAN, J.; DRUILHET, A. Observations and Modeling of Downward Radiative Fluxes (Solar and Infrared) in Urban/Rural Areas. Journal of Climate and Applied Meteorology, v. 22, p. 134-142, 1983. http://dx.doi.org/10.1175/1520-0450(1983)022\%3C0134:OAMODR\%3E2.0.CO;2 
FARR, T. G.; ROSEN, P. A.; CARO, E.; CRIPPEN, R.; DUREN, R.; HENSLEY, S. et al.The Shuttle Radar Topography Mission. Reviews of Geophysics, v. 45, p. RG2004, 2007.

FAUSTO, M. A.; MACHADO, N. G.; NOGUEIRA, J. S.; BIUDES, M. S. Net radiation estimated by remote sensing in Cerrado areas in the Upper Paraguay River Basin. Journal of Applied Remote Sensing, v. 8, p. 1-17, 2014. http://dx.doi.org/10.1117/1.JRS.8.083541

FEARNSIDE, P. M. Hidroelétricas na Amazônia: impactos ambientais e sociais na tomada de decisões sobre grandes obras. Manaus: Editora do INPA, 2015. 288 p.

FREY, C. M.; RIGO, G.; PARLOW, E. Urban radiation balance of two coastal cities in a hot and dry environment. International Journal of Remote Sensing, v. 28, n. 12, p. 26952712, 2007. http://dx.doi.org/10.1080/01431160600993389

GARTLAND, L. Ilhas de Calor: Como mitigar zonas de calor em áreas urbanas. Tradução Silvia Helena Gonçalves. São Paulo: Oficina de Textos, 2010. 248 p.

GOMES, L. C. F.; SANTOS, C. A. C.; ALMEIDA, H. A. Balanço de energia à superfície para a cidade de Patos-PB usando técnicas de sensoriamento remoto. Revista Brasileira de Geografia Física, v. 6, n. 1, p. 015-028, 2013.

GRIMMOND, C. S. B.; OKE, T. R. Aerodynamic properties of urban areas derived from analysis of surface form. Journal of Applied Meteorology, v. 38, p. 1262-1292, 1999. http://dx.doi.org/10.1175/1520-0450(1999)038\%3C1262:APOUAD\%3E2.0.CO;2

INSTITUTO BRASILEIRO DE GEOGRAFIA E ESTATÍSTICA - vpn. Mapa de Biomas e de Vegetação. 2004. Disponível em:

<http://www.ibge.gov.br/home/presidencia/noticias/21052004biomashtml.shtm>.

Acesso em: 25 ago. 2015

LIBERATO, A. M. Albedo à superfície a partir de imagens LANDSAT 5 - TM em áreas de floresta e pastagem na Amazônia. Revista de Geografia (UFPE), v. 28, n. 1, p. 110$119,2011$.

LOPES, L. Estudo do conforto térmico de uma praça e um bosque na cidade de Humaitá/AM. 2013. Trabalho de Conclusão de Curso (Graduação em Engenharia Ambiental) - Universidade Federal do Amazonas, Manau, 2013.

NASCIMENTO, F. C. A.; ARAÚJO, F. R. C. D.; SANTOS, C. A. C.; SANTOS, E. G. Análise das mudanças ambientais provocadas pela expansão urbana na cidade de Mossoró-RN, através do uso de técnicas de Sensoriamento Remoto. Revista Brasileira de Geografia Física, v. 7, n. 4, p. 636-642, 2014.

OKE, T. R.; SPRONKEN-SMITH, R. A.; JAHUREGUI, E.; GRIMMOND, C. S. B. The energy balance of central Mexico City during the dry season. Atmospheric Environment, v. 33, p. 3919-3930, 1999. http://dx.doi.org/10.1016/S13522310(99)00134-X

OLIVEIRA, L. M. M.; MONTENEGRO, S. M. G. L.; ANTONINO A. C. D.; SILVA, B. B.; MACHADO, C. C. C.; GALVÍNCIO, J. D. Análise quantitativa de parâmetros biofísicos de bacia hidrográfica obtidos por sensoriamento remoto. Pesquisa Agropecuária Brasileira, v. 47, p. 1209-1217, 2012. http://dx.doi.org/10.1590/S0100204X2012000900005 
PEREIRA, G.; MORAES, E. C.; ARAI, E. OLIVEIRA, L. G. L. Estudo preliminar da estimativa da reflectância e albedo de microssistemas pantaneiros a partir de imagens de satélite. Revista Brasileira de Cartografia, n. 59, n. 1, p. 55-61, 2007.

PONZONI, F. J.; SHIMABUKURO, Y. E.; KUPLICH, T. M. Sensoriamento remoto da vegetação. São Paulo: Oficina de Textos, 2012. 160 p.

QUERINO, C. A. S.; MOURA, M. A. L.; LYRA, R. F. F.; MARIANO, G. L. Avaliação e comparação de radiação solar global e albedo com ângulo zenital na região Amazônica. Revista Brasileira de Meteorologia, v. 21, n. 3a, p. 42-49, 2006.

SILVA, B. B.; SILVA, S. T. A.; GOMES, H. B. Balanço de radiação no perímetro irrigado São Gonçalo-PB mediante imagens Orbitais. Revista Caatinga, v. 24, n. 3, p. 143-152, 2011.

SOARES-FILHO, B. S.; NEPSTAD, D. C.; CURRAN, L.; CERQUEIRA, G. C.; GARCIA, R. A.; RAMOS, C. A. et al. Cenários de desmatamento para Amazônia. Estudos Avançados, v. 19, n. 54, p. 138-152, 2005.

VIDOTTO, E.; PESSENDA, L.C. R.; RIBEIRO, A. S.; FREITAS, H. A.; BENDASSOLLI, J. A. Dinâmica do ecótono floresta-campo no sul do estado do Amazonas no Holoceno, através de estudos isotópicos e fitossociológicos. Acta Amazônica, v. 37, n. 3, p. 385 400, 2007. http://dx.doi.org/10.1590/S0044-59672007000300010

Von RANDOW, C.; MANZI, A. O.; KRUIJT, B.; OLIVEIRA, P. J.; ZANCHI, F. B.; SILVA, R. L.et al. Comparative measurements and seasonal variations in energy and carbon Exchange over Forest and pasture in South West Amazonia. Theoretical and Applied Climatology, v. 78, p. 5-26, 2004. http://dx.doi.org/10.1007/s00704-004-0041-z

ZANCHI, F. B.; WATERLOO, M. J.; AGUIAR, L. J. G.; VON RANDOW, C.; KRUIJT, B.; CARDOSO, F. L. et al. Estimativa do Índice de Área Foliar (IAF) e Biomassa em pastagem no estado de Rondônia, Brasil. Acta Amazônica, v. 39, n. 2, p. 335-348, 2009. http://dx.doi.org/10.1590/S0044-59672009000200012 